\title{
Compter les morts aux frontières : des contre- statistiques de la société civile à la récupération (inter)gouvernementale
}

Counting Migrant Deaths at the Borders: From Civil Society's Counter-statistics

to (Inter)governmental Recuperation

Contabilizar los muertos en las fronteras: de las contra-estadísticas de la sociedad civil a la recuperación (inter)gubernamental

Charles Heller et Antoine Pécoud

\section{(2) OpenEdition}

Journals

Édition électronique

URL : https://journals.openedition.org/remi/8732

DOI : $10.4000 /$ remi.8732

ISSN : $1777-5418$

Éditeur

Université de Poitiers

Édition imprimée

Date de publication : 1 septembre 2017

Pagination : 63-90

ISBN : 979-10-90426-59-7

ISSN : 0765-0752

Référence électronique

Charles Heller et Antoine Pécoud, « Compter les morts aux frontières : des contre-statistiques de la société civile à la récupération (inter)gouvernementale », Revue européenne des migrations

internationales [En ligne], vol. 33 - n² et 3 | 2017, mis en ligne le 01 septembre 2019, consulté le 14 avril 2022. URL : http://journals.openedition.org/remi/8732 ; DOI : https://doi.org/10.4000/remi.8732 


\title{
Compter les morts aux frontières : des contre-statistiques de la société civile à la récupération (inter)gouvernementale
}

\author{
Charles Heller ${ }^{1}$ et Antoine Pécoud ${ }^{2}$
}

\section{Introduction}

Depuis les soulèvements dans le monde arabe et les conflits qui secouent cette région du monde, la zone euro-méditerranéenne vit au rythme des naufrages en mer, et des milliers de migrants qui perdent la vie au large des côtes européennes. L'attention médiatique et politique suscitée par la " crise des migrants " (ou " crise des réfugiés ") a contribué à rendre visible - voir à spectaculariser - un phénomène déjà ancien : les " morts aux frontières " ne constituent pas, en effet, un phénomène récent ; ils sont de longue date une des conséquences du conflit de mobilité qui oppose les politiques de contrôle des États les plus riches au désir de migrer des populations du Sud, auxquelles on dénie le droit de franchir légalement les frontières. Si ces décès ont longtemps été peu documentés, ils n'en ont pas moins fait l'objet, depuis le début des années 1990, de l'attention de la société civile : plusieurs associations ont alors entamé un décompte de ces morts, avec l'intention de rendre visible les conséquences humaines de la "Forteresse Europe ", de demander des comptes aux États européens et d'appeler à une réorientation des politiques de fermeture des frontières.

Dans le climat de crise actuel, ces initiatives de la société civile ont fait des émules et ont inspiré d'autres acteurs, à commencer par l'Organisation internationale pour les migrations (OIM). En 2013, cette dernière a lancé le Missing Migrant Project (MPP) ${ }^{3}$, dont l'objectif est de fournir des données statistiques

\footnotetext{
1 Chercheur associé au Centre de recherche en architecture, Goldsmiths, Université de Londres et post-doctorant soutenu par le Fonds National de la Recherche Suisse (FNS) au Centre, pour l'Étude des Migrations de I'Université Américaine du Caire ainsi qu'au Centre d'Études et de Documentation Économiques, Juridiques et Sociales du Caire, Centre for Research Architecture, Department of Visual Cultures, Goldsmiths College, University of London, 8 Lewisham Way, New Cross, London SE14 6NW, UK ; chazheller@yahoo.com

2 Professeur de sociologie, Université Paris 13 et chercheur associé au CERI/Sciences Po, UFR DSPS, Université Paris 13, 99 avenue J.-B. Clément, 93430 Villetaneuse ; antoine.pecoud@univ-paris13.fr

Cet auteur remercie la Fondation des sciences sociales pour son soutien.

3 Cf. http://missingmigrants.iom.int/ (consulté le 27/09/2016).
} 
sur les décès et les disparitions de migrants. Ce projet consiste notamment en un site Internet, qui permet d'obtenir " en temps réel " des informations sur ces morts : sont ainsi renseignés le nombre de migrants concernés, leur pays d'origine, le lieu et les raisons de leur décès (noyade, hypothermie, accidents, etc.), leur âge et leur sexe, ainsi que la route migratoire qu'ils étaient en train d'emprunter. Toutes les régions du monde sont concernées, même si - comme I'indique I'OIM elle-même sur son site ${ }^{4}$ - cette initiative répond avant tout à la situation dans la zone euro-méditerranéenne.

Au travers d'une analyse du Missing Migrant Project de I'OIM, cet article propose une réflexion sur les enjeux politiques soulevés par les morts aux frontières et en particulier sur la manière dont ces décès sont pris en compte par une organisation intergouvernementale dont une des fonctions est précisément d'aider les États à mieux contrôler leurs frontières. II repose sur le constat suivant : les morts aux frontières résultent d'une prise de risque par les migrants, laquelle est largement liée à l'impossibilité d'obtenir un accès légal à la migration ; l'intensification et la diversification, par l'Union européenne et les pays européens, de leurs méthodes de contrôle des frontières accroissent donc directement la précarité et les dangers de l'expérience migratoire.

Ce rapport de causalité entre surveillance des frontières et décès de migrants ne signifie pas nécessairement que les États sont juridiquement responsables de ces décès, ni qu'ils aient l'intention de les provoquer; il suffit cependant pour établir le rôle direct des États dans les conditions qui conduisent les migrants à prendre des risques souvent mortels. Ce rôle central joué par les États européens n'est par ailleurs pas incompatible avec la reconnaissance du rôle d'autres acteurs, comme les "passeurs ": les gouvernements des pays de destination blâment régulièrement ces intermédiaires, qui organiseraient pour leur seul profit ces traversées de la Méditerranée, sans tenir compte des risques liés à la piètre qualité des embarcations ou aux conditions météorologiques défavorables.

Or, I'appropriation du décompte des morts aux frontières par une organisation intergouvernementale comme I'OIM transforme profondément la dimension politique de cette pratique. En effet, alors que la société civile compte les morts pour s'opposer aux politiques des États qui conduisent les migrants à prendre des risques pour accéder à leur territoire, I'OIM participe elle-même au contrôle des migrations. Elle joue un rôle important dans les politiques migratoires des pays occidentaux, et notamment dans leurs efforts pour contrôler leurs frontières au travers de stratégies déterritorialisées (ou externalisées). Si un des slogans de cette organisation est de promouvoir une migration " humaine et ordonnée pour le bénéfice de tous ${ }^{5}$, les recherches empiriques sur ses projets ont établi qu'elle est un acteur dans la mondialisation du contrôle des migrants : en s'impliquant, par exemple, dans l'introduction de nouvelles technologies de contrôle dans les zones de départ et de transit, dans le renvoi (parfois qualifié de " retour volontaire ") de migrants dans leur pays d'origine, ou dans des campagnes d'information pour dissuader les migrants potentiels de partir, l'OIM s'aligne sur les préoccupations sécuritaires des États de destination

4 Cf. https://missingmigrants.iom.int/about

5 Cf. https://www.iom.int/fr/propos-de-loim 
et renforce leur capacité de contrôle ${ }^{6}$. Elle contribue donc à créer les conditions qui conduisent aux morts aux frontières, c'est-à-dire précisément à cette réalité qu'elle prétend documenter et combattre dans son Missing Migrant Project.

C'est ainsi que I'OIM a lancé, le 10 juin 2016, un projet de " renforcement des capacités " des gardes-côtes en Libye ; grâce à un financement du gouvernement des Pays-Bas, I'OIM souhaite " sauver des vies en mer " en formant les fonctionnaires libyens et en leur fournissant du matériel ${ }^{7}$. Or, quatre jours après I'annonce de ce projet, le 14 juin 2016, Amnesty International dénonçait les pratiques des gardes-côtes en Libye : I'organisation de défense des droits de I'homme révélait que des migrants ont été l'objet de tirs lors de leur interception en mer, avant d'être emprisonnés dans des centres de détention et d'y être exposés à la torture ${ }^{8}$. Comme le documente par ailleurs Brachet (2016), I'OIM joue donc un rôle assez ambigu en Libye : tout en prétendant poursuivre des objectifs humanitaires dans un pays en pleine guerre civile, elle travaille à empêcher les migrants de tenter la traversée afin de gagner l'Europe.

II y a ainsi une tension interne aux activités de l'OIM, qui compte et déplore les morts aux frontières tout en contribuant à créer les conditions qui conduisent à ces décès. L'objectif de cet article n'est cependant pas seulement de mettre en évidence cette ambiguïté ou de polémiquer sur l'hypocrisie, voire le cynisme, de cette organisation. Nous souhaitons avant tout comprendre les raisons qui poussent I'OIM à recenser les morts aux frontières, la manière dont cette activité s'insère dans ses stratégies et ses discours, et interroger les conséquences de son implication récente dans une pratique qui était, jusqu'il y a peu, essentiellement conduite par des ONG.

L'article est structuré de la façon suivante. Il propose tout d'abord un cadre théorique pour penser le rapport entre statistique, États, migrations, et société civile. Il décrit ensuite le phénomène des " morts aux frontières " ainsi que les différentes initiatives citoyennes qui ont tenté de les documenter. II analyse la reprise, par I'OIM, de cette activité de comptage des morts et montre qu'elle $s^{\prime}$ inscrit dans un phénomène plus large $d^{\prime}$ " humanitarisation des frontières " (Cuttitta, 2014 ; Walters, 2011), qui voit le contrôle des frontières être justifié non seulement par des raisons d'ordre sécuritaire, mais aussi par des considérations humanitaires. Finalement, nous analysons l'implication de la récupération par I'OIM du comptage des morts aux frontières pour les acteurs de la société civile, et explorons la manière dont ces derniers peuvent tenter de poursuivre leur critique des politiques migratoires dans ce nouveau contexte.

6 Voir par exemple Andrijasevic et Walters (2011), Georgi (2010) et Pécoud (2017).

$7 \mathrm{Cf}$. https://www.iom.int/news/netherlands-iom-build-libyan-coast-guard-capacity-savelives-sea (consulté le 27/09/2016).

8 Cf. https://www.amnesty.org.uk/press-releases/refugees-shot-libyan-coastguard-beingdetained-shocking-conditions-back-libya\#.V1-YY71NHQU.twitter (consulté le 27/09/2016). 


\section{États, (contre-)statistiques et migrations}

Dans son cours de 1978 au Collège de France, intitulé Sécurité, Territoire, Population, Foucault (2004) évoque à plusieurs reprises les statistiques et leur rôle fondamental dans une transformation du pouvoir au cours du XVIle siècle, c'est-à-dire dans la naissance de ce qu'il nomme "gouvernementalité ". Cette dernière est décrite comme une forme de pouvoir « qui a pour cible la population, pour forme majeure de savoir l'économie politique, pour instrument technique essentiel les dispositifs de sécurité "(Foucault, 2004 : 111). La gouvernementalité suppose un changement dans la production du savoir, car elle requiert une évaluation constante des forces et des ressources de l'État. L'outil principal pour produire ce savoir est la statistique - terme dont l'étymologie renvoie d'ailleurs à la connaissance de l'État. C'est ainsi que l'État se soucie de sa population, en termes de quantité, mortalité, natalité, sexualité, richesses, etc.

Dans un ouvrage classique, Scott (1998) s'inspire de Foucault et démontre le lien consubstantiel entre État et statistique : grâce aux statistiques, les États " voient " leur territoire et leur population et, par là même, les gouvernent. Les statistiques introduisent une relation productive et circulaire entre État, population, savoir et gouvernement. Dans une optique constructiviste, elles " créent " un État ou un pays en produisant un territoire et une population homogènes. Faute de données statistiques, territoire et population ne seraient pas perçus comme un tout, mais comme une collection hétéroclite d'éléments sans cohérence ni continuité. De plus, la production même de ce savoir statistique est liée au gouvernement de la population, dans la mesure où les statistiques sont générées au travers d'un appareil administratif qui en retour opère sur la base de ce savoir. Les statistiques, comme forme de savoir, sont donc inextricablement liées aux rapports de pouvoirs (voir aussi Legg, 2005 ; Rose-Redwood, 2012).

Les migrations offrent un exemple clair de ce lien. Les statistiques en la matière ne décrivent pas simplement (ou " objectivement ") une réalité sociale préexistante. Les statistiques contribuent au contraire à faire exister les migrations, en les rendant visibles et chiffrables par les États ; ce faisant, elles sont à la fois le produit des politiques migratoires, et un des outils privilégiés par lesquels ces politiques opèrent. Par exemple, au cours du XIXe siècle, c'est la pratique du recensement et la collecte de statistiques qui ont contribué à l'émergence de la catégorie du " migrant " et à une différenciation entre la population " nationale " et étrangère. Le contexte actuel fournit de nombreux exemples de ce lien entre statistiques et gouvernement des migrations. Pour se limiter à la thématique de cet article, les gouvernements utilisent les données statistiques pour construire les migrations transméditerranéennes comme une menace qui doit être combattue. Parmi les nombreux acteurs qui visent à la fois à quantifier et à gouverner ces formes illégalisées de mobilité, I'agence Frontex produit des " analyses de risque " qui décrivent l'évolution (chiffrée) de la situation aux frontières extérieures de I'UE, ce qui en retour justifie ses activités en termes d'interventions et d'interceptions de migrants (Stenum, 2012 ;Tazzioli, 2015). 
Figure 1 : Page de couverture du rapport 2011 de Frontex montrant la photographie d'une embarcation chargée de migrants pendant son interception

$$
\text { હ FRONTEX }
$$

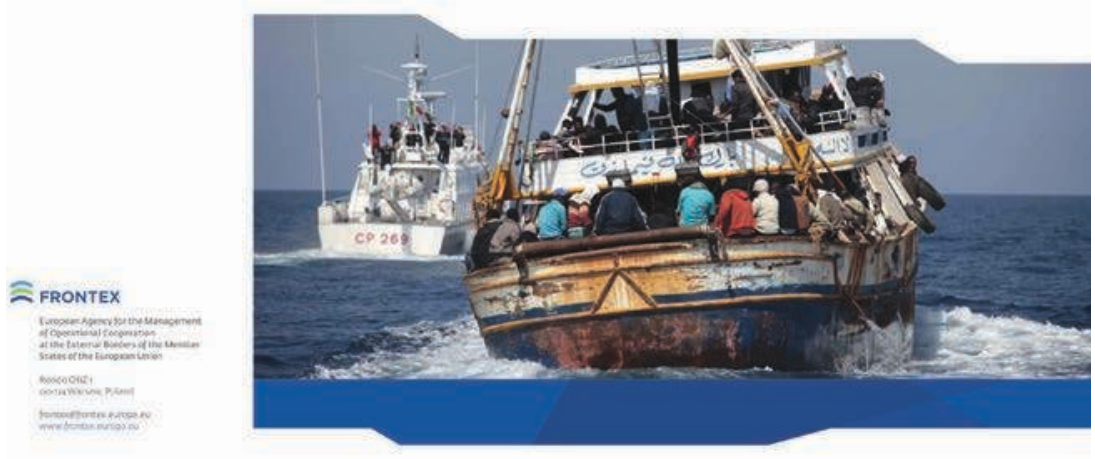

\section{General Report 2011}

Source : Frontex (2011) General Report 2011, Warsaw, Frontex, 60 p.

Figure 2 : Statistiques de Frontex concernant les interceptions aux trois " routes " principales à travers la Méditerranée

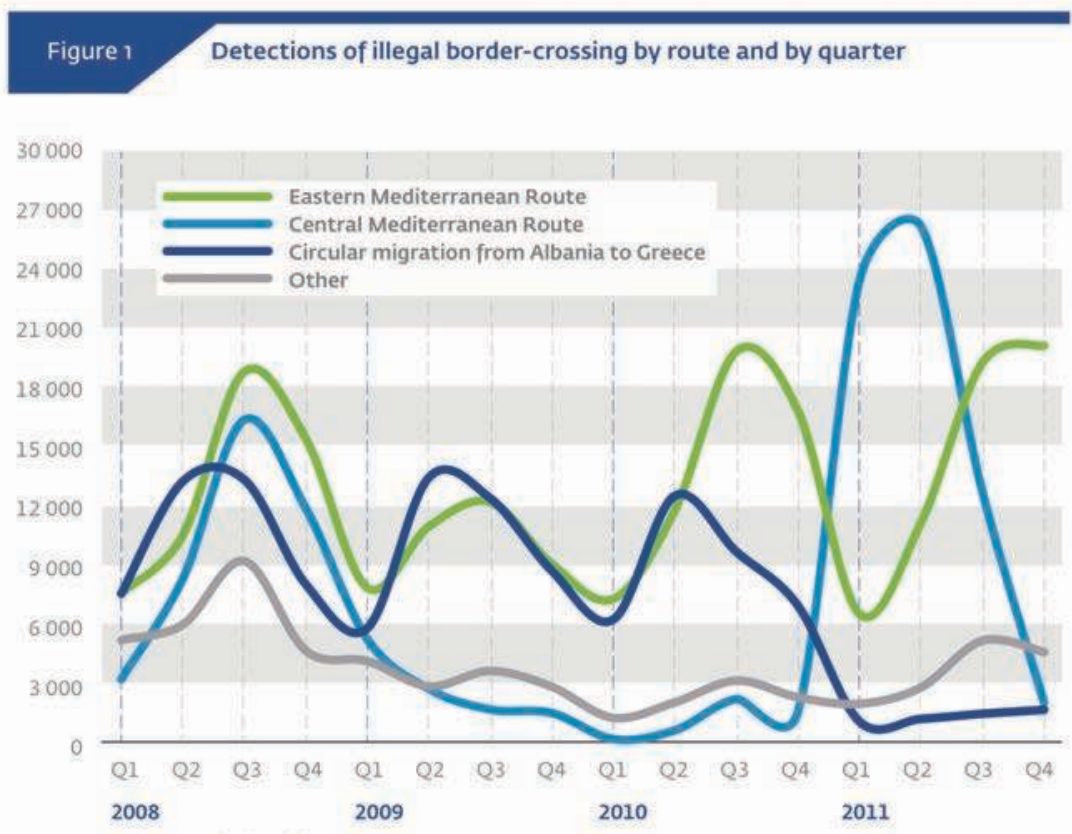

Source: FRAN data received as of 10 February 2012

Source : Frontex (2012) Annual Risk Analysis 2012, Warsaw, Frontex, 64 p. 
Les statistiques d'interceptions publiées par Frontex, dont la visualisation même fait écho aux discours sur les "vagues " de migrants arrivant sur les côtes de l'Europe, participent à la production de ce que De Genova (2013) a appelé le "spectacle des frontières ". Ces graphiques sont l'équivalent, en termes statistiques, des photos d'embarcations surchargées de migrants. Ces statistiques quantifient une " menace " qui n'est mesurée (et mesurable) qu'au moment de sa neutralisation par les gardes-frontières. À travers ces chiffres, c'est donc simultanément la menace de la migration et le contrôle opéré par les États européens qui sont rendus visibles. Mais comme le note de Genova, cette " spectacularisation " de l'instant de l'interception rend d'autres aspects de la question invisibles, comme les politiques qui illégalisent les migrations ou les formes de précarité et d'exploitation qui attendent les migrants à l'intérieur des pays européens.

Les analyses inspirées de Foucault permettent également de comprendre comment les statistiques peuvent être (ré)appropriées pour contester les pratiques de gouvernement. Foucault souligne en effet le rôle des résistances au pouvoir dans la pratique du pouvoir - ou comment pouvoir et résistance au pouvoir sont co-constitutifs I'un de l'autre (Foucault, 2004 : 198-199). Rose (1999) montre ainsi que, dans les sociétés démocratiques, les États ont été amenés à divulguer une partie des données statistiques dont ils disposent sur leur propre population, ce qui permet à leurs citoyens de juger et de critiquer leurs politiques. Si les États produisent des statistiques pour mesurer et évaluer leur action, la diffusion de ces statistiques génère en retour ce que Rose nomme des " citoyens calculant " (calculating citizens), qui utilisent les chiffres de l'État pour demander des comptes aux autorités. Dans la même optique, Bruno et al. (2014) proposent le terme de " statactivisme " pour désigner l'usage critique des chiffres : ils démontrent que, de même qu'elles sont liées à l'exercice du pouvoir, les statistiques sont également liées aux pratiques de contestation et d'émancipation ; en particulier, les statistiques sont adaptées à la remise en cause de ce qu'ils appellent la gouvernementalité néolibérale, car cette dernière se caractérise par un recours obsessionnel à l'évaluation chiffrée - ce qui l'expose à une critique émanant de ces mêmes chiffres.

Cet usage critique des statistiques s'inscrit dans ce que Foucault (2004 : 199) appelle des " contre-conduites " et nous proposons donc de les qualifier de " contre-statistiques ${ }^{9}$. C'est dans cette perspective qu'on peut analyser la manière dont des acteurs de la société civile ont de longue date procédé au décompte des morts aux frontières. Ces chiffres apparaissent comme la contrepartie critique aux données collectées et publiées par les États sur les flux migratoires et les traversées des frontières. L'objectif des acteurs à l'œuvre derrière ce décompte n'est pas de gouverner les migrations, mais de rendre visibles les effets mortifères des politiques migratoires imposées par les États - et de chiffrer ces effets pour mieux les contester.

9 Ailleurs, nous avons également qualifié ces pratiques de " statistiques tactiques " pour souligner leur positionnement tactique dans le champ de pouvoir (Heller, 2015). 


\section{Les " morts aux frontières " : la production de contre-statistiques non gouvernementales}

Les décès liés au processus de la migration sont probablement aussi anciens que les migrations elles-mêmes. Une généalogie dépasse de loin le cadre de cet article, mais devrait suivre un chemin aussi sinueux et mouvant que les trajectoires des migrants à travers I'histoire, et disséquer les différentes formes de violences auxquelles ils ont été soumis. II conviendrait de rappeler la migration forcée qu'a constitué l'esclavage transatlantique, durant lequel au moins deux millions d'esclaves sont morts lors du " noir passage " que représentait la traversée de l'océan (Blackburn, 2011), ou encore les migrants chinois qui mourraient en Californie à la fin du XIXe siècle en tentant d'échapper au Chinese Exclusion Act de 1882 (Nevins, 2003). Dans la phase de migration qui a suivi la Seconde Guerre mondiale, et qui a notamment vu la migration d'anciens peuples colonisés vers les centres de l'économie mondiale, des décès ont été répertoriés de manière structurelle à chacune des zones de fracture séparant les régions les plus riches des régions les plus pauvres du globe, et ce à partir du moment où les régions les plus fortunées ont tenté de se protéger des populations jugées " indésirables " (Agier, 2008).

On est donc en présence d'un phénomène ancien, durable et global, qui constitue un aspect incontournable des dynamiques migratoires. Comme suggéré dans l'introduction, le déni d'un accès légal à un territoire conduit à des formes clandestines de franchissement des frontières. Privés de l'accès à des moyens de transport formels (qui pourtant existent abondamment aujourd'hui), les migrants qui refusent cette " assignation à résidence " doivent recourir à des passeurs ainsi qu'à des moyens de transport dangereux et précaires. Le franchissement d'une frontière sans autorisation est l'occasion d'une périlleuse exposition des corps des migrants à des menaces de nature variée, qui vont de la privation de liberté par des passeurs aux tirs de gardes-frontières, en passant par les éléments naturels inhospitaliers qui caractérisent les mers ou les déserts. Ainsi, les zones de friction et de mort les plus importantes se situent à la frontière entre le Mexique et les USA, au large de l'Australie, dans les Caraïbes, dans la Corne de l'Afrique, en Asie du Sud-est, en Afrique du Sud ou encore au cœur du Sahara. Mais c'est la région euro-méditerranéenne qui est cependant devenue aujourd'hui le principal cimetière de migrants au monde (Clochard et Lambert, 2015).

De plus, les formes de violences auxquelles sont soumis les migrants ne s'arrêtent pas aux frontières. De manière croissante, et selon les termes mêmes des tenants du " management intégré des frontières " (integrated border management), le contrôle s'exerce " avant, à travers, à et après la frontière ". C'est donc l'ensemble des trajectoires migratoires qui s'en trouvent précarisées. Pour reprendre un concept des critical border studies, le contrôle de la frontière territoriale interétatique se double de "pratiques de la frontière " (bordering practices), qui se matérialisent partout où les migrants rencontrent les fonctions d'inclusion/exclusion des États (Newman, 2006). Dans cette perspective, les morts aux frontières peuvent survenir en mer Méditerranée ou dans le Sahara, mais aussi dans des centres de détention, lors d'expulsions, sur les lieux de travail des migrants précarisés, et même suite à des crimes racistes ou au suicide de migrants accablés par leurs conditions de vie. Tous ces types de décès 
relèvent d'une frontière étendue, tant spatialement que socialement. Weber et Pickering (2011) parlent de "violence structurelle " pour désigner la manière dont les morts aux frontières sont la conséquence d'une violence multiforme, perpétrée par des acteurs multiples et par conséquent difficiles à cerner, mais dans laquelle les politiques des États jouent un rôle surdéterminant (voir aussi Albahari, 2015 ; Pezzani, 2015).

Structurelles, les morts aux frontières passent néanmoins souvent inaperçues, du moins du grand public. Les migrants meurent en général dans des endroits peu accessibles (mer, déserts) ou dans des lieux périphériques, voire fermés. Beaucoup d'entre eux voyagent clandestinement, et meurent tout aussi clandestinement. Même lorsque ces décès font l'objet d'une publicité médiatique, ils suscitent souvent une indifférence mêlée de résignation : les victimes sont des ressortissants de pays du Sud et, dans un monde inégalitaire et postcolonial, leur vie ne semble pas avoir la même valeur que celles des citoyens des pays développés (Van Houtum et Boedeltje, 2009). Quand bien même ces morts sont déplorées, elles sont souvent perçues comme un " mal nécessaire ", inhérent au contrôle des migrations.

De plus, alors que les États documentent méticuleusement l'état de leur propre population ainsi que l'entrée sur leur territoire de citoyens étrangers, ils n'ont accordé que très peu d'attention dans leur pratique statistique aux personnes mortes en tentant de traverser leurs frontières. Comme l'écrit Kobelinsky (2015: 200), " alors que des moyens colossaux sont dépensés par I'Union européenne afin de renforcer la surveillance aux frontières extérieures, aucune ligne budgétaire n'est prévue pour la gestion des morts de la migration ". $C^{\prime}$ est là le corolaire de la visibilité par les statistiques: les phénomènes qui ne sont pas documentés sont condamnés à l'invisibilité ; les États ne " voient " que ce qu'ils chiffrent et nombre d'enjeux demeurent ainsi inexistants, non parce qu'ils ne sont pas problématiques, mais parce qu'ils sont (parfois délibérément) relégués dans l'ombre. En négligeant de recenser les morts aux frontières, les États condamnent ces morts à l'invisibilité.

Certes, ces morts surviennent de façon visible : des corps échouent sur des plages, des bateaux chavirent et sont secourus. Mais faute de statistiques, les morts aux frontières ne seraient rien d'autre que des décès fortuits, connus des seuls habitants des rivages concernés ou de quelques rares spécialistes. Les statistiques permettent d'agréger ces décès, de considérer d'une même façon des événements de différentes natures (noyades, déshydratation, etc.), survenant dans des lieux différents et éloignés les uns des autres. Elles construisent un tout, un phénomène global. C'est pour cette raison que les acteurs de la société civile collectent des " contre-statistiques " sur les morts aux frontières. Les objectifs sont multiples : garder une trace de ces morts souvent sans noms ni visages, rendre visible ce phénomène dans sa globalité, contrer l'indifférence et, in fine, contester les politiques migratoires qui les fabriquent (Blanchard et al., 2008). Ces efforts ne sont pas sans précédent : les militants anti-esclavagistes des XVIIle et XIXe siècles tentaient de sensibiliser les populations des métropoles à l'expérience et à la souffrance des esclaves, afin qu'elles n'achètent pas les produits souillés de sang, et ce notamment à travers des statistiques des morts pendant la traversée de l'Atlantique (Perry, 2012 ; Heller, 2015). 
Dès 1993, I'organisation United for Intercultural Action commence à publier une liste des morts (List of deaths) ${ }^{10}$, qui recense dans sa dernière version (juin 2017) 33305 « morts documentées de requérants d'asile, réfugiés et migrants à cause des politiques restrictives de la Forteresse Europe $"{ }^{11}$ La définition retenue est large, puisque sont inclus tous les décès qui, d'une manière ou d'une autre, peuvent être liés à cette " forteresse ". Cela comprend des décès tout au long des trajectoires des migrants, ainsi que des cas de suicides, de violences policières, de morts post-expulsion, ou suite à l'absence de soins médicaux. Le recensement, compilé dans une liste très détaillée et comprenant de nombreuses catégories, est effectué sur la base de cas rapportés par des journaux, mais aussi par les ONG et par les militants participant à ce projet.

Depuis 2006, le blog Fortress Europe recense également les décès entre I'Europe et l'Afrique du Nord depuis 1988 ; bien que moins détaillée, cette base de données est constituée à partir de sources comparables et sur la base d'une conception similaire des morts aux frontières : elle aboutit au chiffre de 27382 décès (entre 1998 et 2016) ${ }^{12}$. Depuis 2012, I'universitaire Thomas Spijkerboer et ses collègues collectent des données dans les registres mortuaires locaux pour créer la base de données Border Deaths ${ }^{13}$. Dans la mesure où tous les corps lors de naufrages documentés ne sont pas retrouvés (et donc enregistrés), cette base de données contient un nombre sensiblement plus bas de décès, à savoir 3188 entre 1990 et 2013. Finalement, entre 2013 et 2016, un réseau de journalistes européens a lancé le projet Migrant Files ${ }^{14}$, qui combinait et fédérait en partie les listes de Fortress Europe et United et est ainsi arrivé à un décompte global plus élevé (plus de 30000 morts depuis 2000).

Il existe donc des estimations assez différentes du nombre de morts aux frontières. Cela n'est guère surprenant, tant ce phénomène est par définition complexe à appréhender : beaucoup de décès ne peuvent être constatés directement, et doivent donc faire l'objet d'estimations par nature incertaines et changeantes. Mais au-delà des difficultés liées à la récolte de l'information, il existe un autre problème, lié à l'absence de définition du concept même de morts aux frontières. Toute activité statistique suppose en effet des catégories ; mais la catégorie " mort aux frontières " ne fait pour l'heure l'objet d'aucune définition standardisée et il est donc possible $d^{\prime} y$ inclure des décès de nature très différente, qui vont des noyades aux suicides de migrants.

Ces incertitudes en termes de définition sont également de nature spatiale et géographique. La société civile tend à adopter une perspective géographiquement extensive. L'essentiel des décès comptés par la société civile surviennent aux frontières maritimes de l'Europe, entre l'Afrique de l'Ouest et les îles Canaries, dans le detroit de Gibraltar et aux frontières des enclaves territoriales espagnoles de Ceuta et Melilla au Maroc, dans le détroit de Sicile (y compris Malte, Lampedusa et les côtes libyennes), en mer Égée (entre Grèce etTurquie),

\footnotetext{
10 Cf. http://www.unitedagainstracism.org/blog/2017/11/10/united-list-of-deaths-to-markthe-day-against-fascism/

11 Cf. www.unitedagainstracism.org/pdfs/listofdeaths.pdf (consulté le 08/07/2017).

12 Cf. http://fortresseurope.blogspot.fr/ (consulté le 27/09/2016).

13 Cf. http://www.borderdeaths.org/ (consulté le 27/09/2016).

14 Cf. http://www.themigrantsfiles.com/
} 
et dans le canal d'Otrante (entre I'Italie et l'Albanie). Mais à cette conception assez classique de la frontière s'ajoutent d'autres lieux, situés à l'intérieur des États : c'est le cas du désert du Sahara (en Algérie et en Libye), des centres de détention aux quatre coins de l'Europe, ou encore des aéroports.

À l'inverse, comme discuté ci-dessous, la manière dont I'OIM définit et conceptualise les " morts aux frontières " est très différente - et plus restrictive, à la fois quant à la nature des décès et à leur localisation. Ces questions de définition sont porteuses d'enjeux très politiques : elles débouchent en effet sur des conclusions très différentes en matière de responsabilités et de mesures à prendre pour remédier au problème. Par exemple, un cas de noyade ou de naufrage peut être attribué à des passeurs, comme l'argumentent régulièrement les États pour conclure que les morts pourraient être évitées grâce à des contrôles plus stricts de la circulation des embarcations transportant des migrants. En revanche, le suicide d'un migrant dans un centre de détention pose des questions toutes autres, relevant de la manière dont les autorités des États concernés traitent les personnes qu'elles prennent en charge.

Au-delà des bases de données qu'elles constituent, ces listes de morts ont acquis une présence dans l'espace public, et ce à travers différents médias et moyens d'expression. En 2004, Le Monde Diplomatique publiait une première carte, réalisée par Olivier Clochard et Philippe Rekacewicz (Migreurop, 2012 : 134-137) et intitulée Des morts par milliers aux portes de l'Europe ${ }^{15}$. Cette carte, régulièrement mise à jour depuis, a elle-même " migré ", circulant dans des rapports, des journaux à grand tirage, des manifestations, des expositions et devenant ainsi un symbole de la violence des frontières. De même, la liste d'United for Intercultural Action a acquis une forte dimension symbolique en étant appropriée et brandie par des militants dans différents contextes, tels que des commémorations ou des manifestations, à l'occasion desquelles elle est imprimée en grand format et devient un objet autour duquel les personnes se rassemblent.

Ainsi, si les États opèrent un regard policier, qui tente de détecter tout franchissement de leurs frontières, les acteurs non gouvernementaux opèrent un regard désobéissant (Heller et Pezzani, 2014), qui n'aspire pas à contrôler la mobilité des migrants, mais à éclairer les conséquences mortifères des politiques des États. Mais si cette schématisation binaire a pu être pertinente, le discours et la collecte des statistiques sur les morts aux frontières ont récemment acquis des significations plus ambiguës, qui ne permettent plus de la situer aisément et exclusivement d'un côté ou de l'autre de cette opposition entre gouvernement et société civile. Pour comprendre ce déplacement, dans lequel s'inscrit notamment le décompte des morts aux frontières par l'OIM, il nous faut comprendre le devenir humanitaire de la frontière.

15 Cf. http://www.monde-diplomatique.fr/cartes/mortsauxfrontieres (consulté le 27/09/2016). 


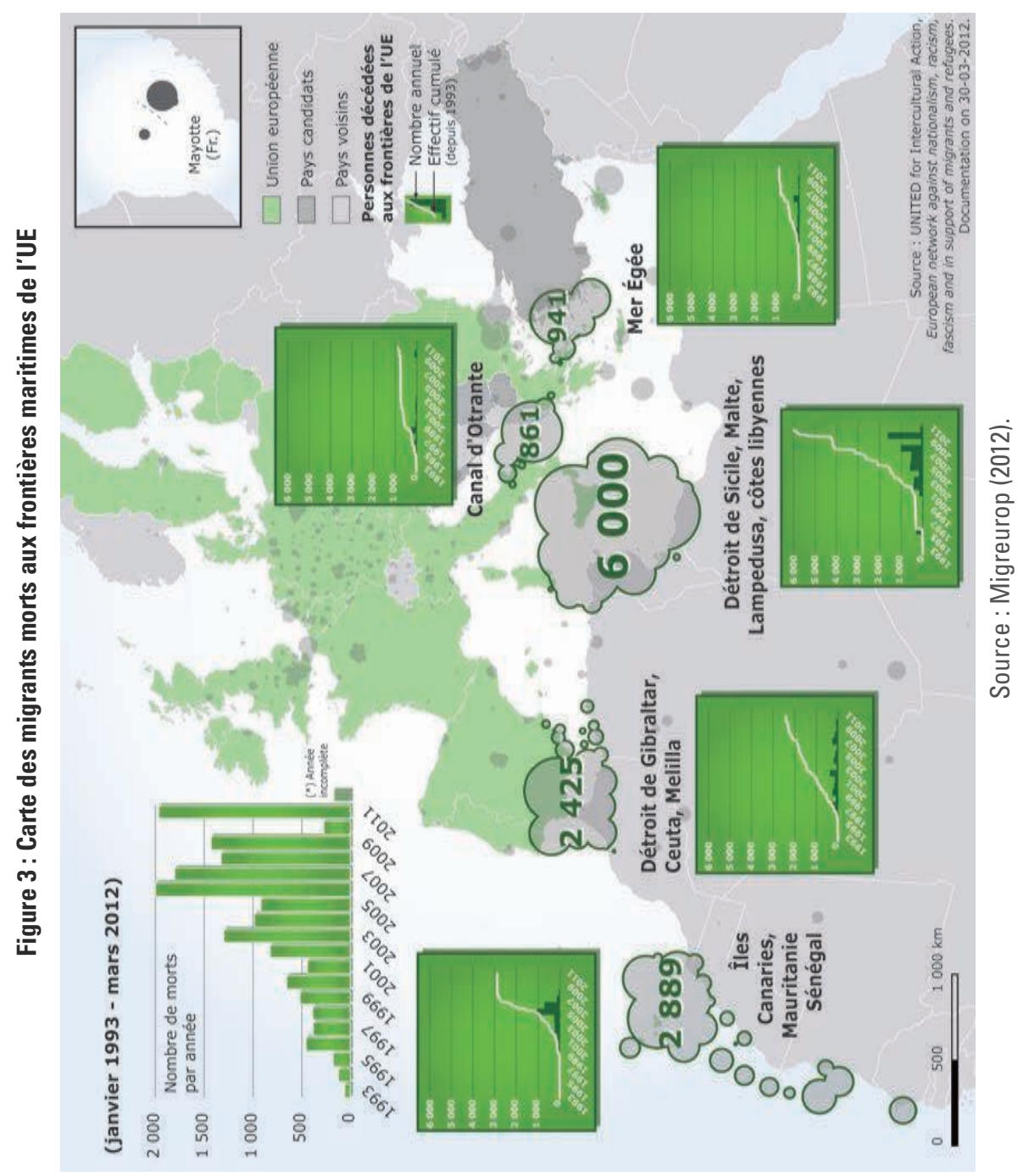


Figures 4 et 5 : Liste des morts d' United for Intercultural Action, imprimée et déroulée devant le Parlement européen lors d'un événement organisé par le Collectif pour une autre politique migratoire (28 avril 2015)

List of 33.305 documented deaths of refugees and migrants due to the restrictive policies of Fortress Europe Documentation as a 15 sine 2017 by UNTEO

Death by Policy - Time for Change!

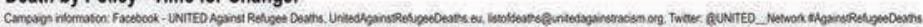

\section{UNIED}

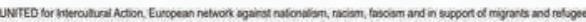

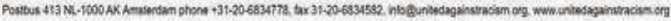

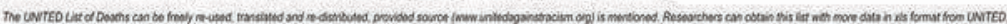

\begin{tabular}{|c|c|c|c|c|c|}
\hline 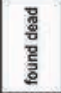 & $\begin{array}{l}\text { ठू } \\
\text { है } \\
\text { है }\end{array}$ & name & region of origin & cause of death & source \\
\hline 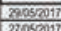 & $\frac{10}{10}$ & N.N. (1 smol chid) & unknown & 2 bodes found, $28 \mathrm{miss}$. & VoAUSNOGS \\
\hline$\frac{27055 \times 17}{2405 \times 17}$ & $\frac{10}{82}$ & NN. & Ininkinoman & 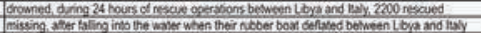 & $\begin{array}{l}\text { Disigabon } \\
\text { USNews }\end{array}$ \\
\hline $2305 / 2017$ & 34 & 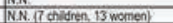 & unkenoma & 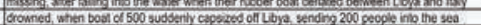 & Dowistarus stewesintive \\
\hline & $\frac{\pi}{2}$ & NN. & Westaticalubiown & 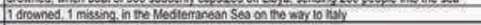 & $\cos$ \\
\hline 22052017 & 2 & N.K.(men) & unknom & Bodies bond hal Mbra (Lbya) & $10 \mathrm{~m}$ \\
\hline 19052017 & 1 & N.N. (30, man) & Mab & 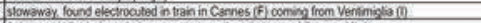 & ParsienNive Mal Onine \\
\hline 180522017 & 2 & N.N. & Unknown & 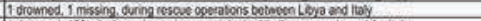 & $10 \mathrm{~m}$ \\
\hline 190550117 & 157 & 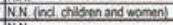 & inknom & 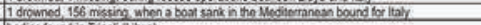 & Lustnlan \\
\hline 17052017 & $\frac{1}{2}$ & NN. & unichom & 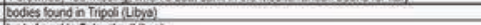 & ion \\
\hline & $i$ & NN. (womsen) & unenewon & 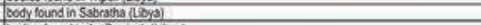 & $10 \mathrm{~m}$ \\
\hline 160520017 & 2 & N.N. I woman 1 man & unknom & 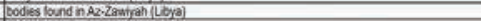 & $10 \mathrm{M}$ \\
\hline 160520017 & 1 & & unknown & cody found in Treoll(Loga) & 100 \\
\hline 140520017 & 7 & N.N.(men) & onknown & 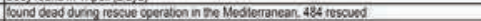 & RTLNisons \\
\hline 07052017 & 163 & N.N (1 boty gatt $9 \mathrm{chn} .40 \mathrm{w}$ & unknowa & 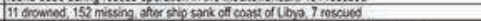 & 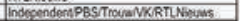 \\
\hline 05052017 & 82 & NN & unknowa & 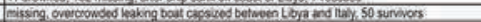 & Indegender PBST Troven \\
\hline 04052017 & 19 & NN & undinown & 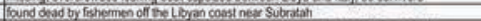 & low \\
\hline 04052017 & 1 & NN & unknown & 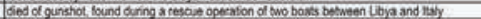 & $10 \mathrm{~m}$ \\
\hline 00052017 & 1 & NNE (man) & whenem & 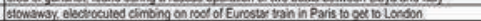 & $\sin$ \\
\hline inMar 17 & 6 & N.N. & uniciom & bound sadu durng a cescue operaton between Loya and liely & 100 \\
\hline $\operatorname{miMy} 17$ & 1 & NN. & whingen & 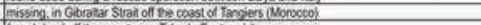 & 100 \\
\hline 26042017 & 1 & NN. & unknown & found daad ott the cosast near Tobrik Eastam Loya & icm \\
\hline 24042017 & 28 & NN. $(2 \mathrm{chn}, 6$ women, 8 men $)$ & Sriacameroon Congo & 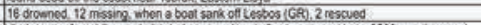 & Rollows 24 Chatica \\
\hline 66042017 & 7 & N.N. B. ber 1 Greg woman & unknoman & & WKFocubDE Ginders \\
\hline 16042017 & 100 & NN. & uningan & 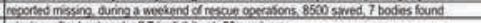 & Moble Eas:Orine \\
\hline 13042017 & 97 & TNN. 15 chidren, 15 wormen] & Sobshomatica & 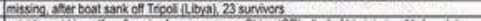 & Merowhordasulvoh \\
\hline 10042017 & 1 & NN. $(29$, man) & Spria & 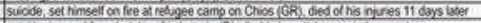 & Ansamed \\
\hline 30032017 & 1 & Faisalmran $\{28$, man & Parsitan & 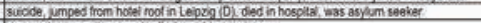 & Rieblebeniceo \\
\hline 229012017 & 5 & NN. & Unenown & 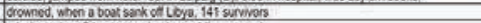 & Reulersillo \\
\hline 23012017 & 240 & NN. & unknown & frissing, when 2 boats capsiad near L Lyan coast & BeCCIVISAn \\
\hline $2 \pi 302017$ & 11 & N.N. 5 chidren) & Spra & 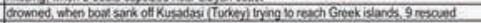 & BBCICT \\
\hline 23032017 & 6 & N.N $(5$ mes, $18.25 \mathrm{y}$ & ARca & 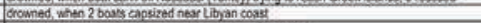 & BBCCTVISA \\
\hline $210 y 2017$ & 68 & NN. & untenom & 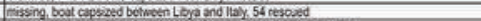 & CTV \\
\hline 0 क्राप्ता & $\frac{1}{1}$ & Mansou Kongate $(33$, man) & Mas & 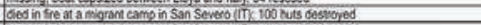 & 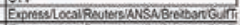 \\
\hline 0220017 & $\frac{1}{1}$ & Nouthou Doumitiag, (36, manj) & Mo & 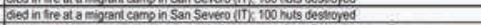 & 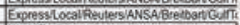 \\
\hline 01032017 & 1 & NN. (man) & Aticas & 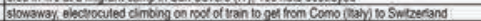 & Wondrado \\
\hline 220020017 & is & NN. & unkenoma & 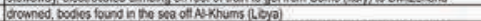 & Rei \\
\hline 200097 & 101 & NN & unknown & 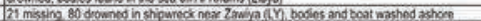 & 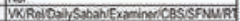 \\
\hline $1802 \times 17$ & 1 & Finedond ofosu (33, man) & Ghana & 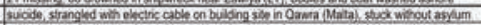 & Timesll \\
\hline $17+702 x+17$ & 1 & NN (man) & uniknom & 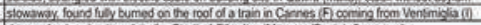 & Percosen \\
\hline 15025017 & 9 & NN: & unknown & 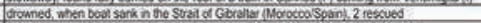 & 100 \\
\hline 120200 & 2 & N.N. & unenown & 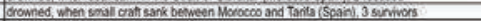 & ICM \\
\hline 04020017 & 1 & N.N. (woman) & Entoges & 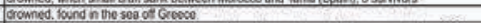 & $\frac{7}{30}$ \\
\hline 35016017 & 1 & NN. (20, man) & Paksten & 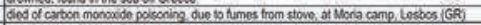 & RoulersiNowsweekKISSouthk \\
\hline 301012017 & $\frac{1}{3}$ & NN. & Urickowa & 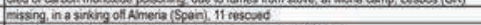 & IOM \\
\hline 280200017 & 1 & NN. $(45, \min )$ & Sinia & 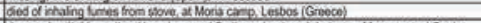 & Rouliers NowsweokKKLSputnk \\
\hline 27012017 & 1 & Ssmuel $16.60 y)$ & $\cos x^{\circ}$ & 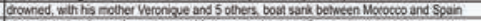 & BeCiReules \\
\hline 24012017 & 1 & NN. $(22, \mathrm{man})$ & Eoge & 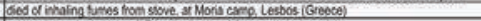 & 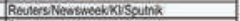 \\
\hline 24012017 & 10 & Nen If ched, I woman, 8 manj & whenom & 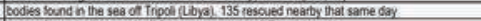 & Ret \\
\hline 210012017 & 6 & & univiom & 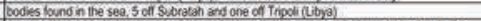 & Res \\
\hline 21012017 & 1 & NN. $(20$, man $)$ & Entiogat & 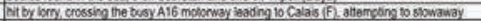 & Theoram Expoess \\
\hline 1710120017 & 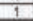 & NN $(\operatorname{man})$ & unknown & 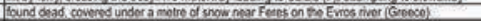 & Sologiobe \\
\hline 140217 & 2 & NN: & uningown & 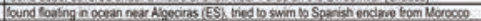 & Indegendects \\
\hline 1400117 & 2 & NN. & uelenoma & 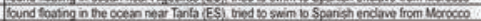 & indigendert \\
\hline $140+177$ & 8 & NN & unknown & 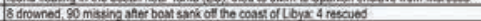 & \\
\hline 14012017 & 1 & N.N. (woma) & $\operatorname{con} e^{\circ}$ & 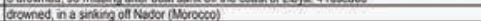 & Sorigiode \\
\hline 14012017 & $\frac{1}{3}$ & N.N. (t moman 2 ment & uniknom & The are of Nssorer Morocos) & Solagiose \\
\hline 16012017 & 3 & NA & unknown & 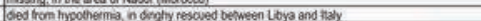 & Solaculoce \\
\hline 14012017 & 1802 & NN & Somala Entre & 8 drowned, 172 missigg ahen boat sank due : & 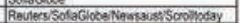 \\
\hline 1302017 & $\frac{1}{2}$ & NN & unknown & consted to seath in a batic croving 191 other passen & hindogendest \\
\hline 13012017 & 21 & 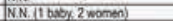 & unknowa & 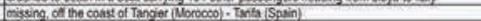 & Solaclootereviles \\
\hline 11012017 & 1 & 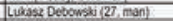 & Poland & 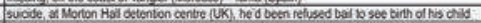 & Opendem Socolisworket \\
\hline 111012017 & $i$ & Veronique (woman) & $\cos n 0^{\circ}$ & ind 5 ohers boal sank between $1 \mathrm{M}$ & BeCReviter \\
\hline H11012017 & 5 & N...(men) & SuosineranAtice & 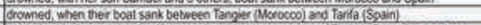 & BBC Reulers \\
\hline 0,0500171 & 1 & 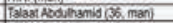 & lag & 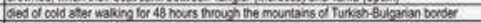 & indesendem \\
\hline 060117 & 1 & Hard Ghabur (29. man) & & 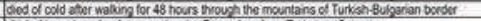 & Indogenders \\
\hline 0300117 & 1 & N.N. $(20, \operatorname{man})$ & Achanistan & 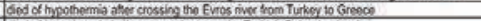 & Independient \\
\hline 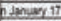 & 1 & N.N. (womin) & Somala & 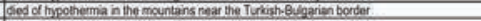 & Indegendert \\
\hline 231236 & 2 & & Substhem Atica & 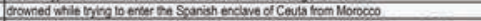 & \\
\hline 251216 & 1 & Non:man) & Kunstatan & 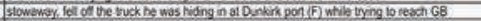 & TMURT \\
\hline $2422 \times 6$ & 1 & N.N. $(17.00 \%$ & $\cos y 0$ & 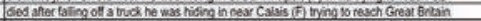 & Veciroungerd \\
\hline 25122016 & 1 & N.N. $(202 . \mathrm{mg} 9)$ & unkionom & 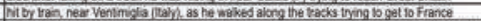 & Patsen \\
\hline 211216 & $\frac{1}{7}$ & NN & unknown & 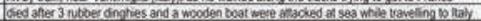 & AEPYYN \\
\hline 161216 & 1 & N.N. $(240$, man $)$ & Mas & umped ous df a migrant wohker centero in Paris when a fro broke oul & AP \\
\hline 0852:16 & 1 & Hai (man) & Somata & 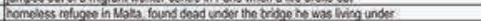 & TimesM \\
\hline $05 \% 1276$ & $\frac{1}{1}$ & 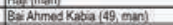 & Sierathogen & 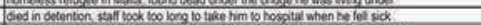 & 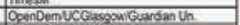 \\
\hline 1220016 & 1 & Trek Chowenury $(64$, man) & Banglacoses & 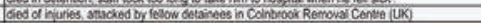 & nogetenderspayan \\
\hline nomec 16 & 4 & N.N. joung man) & paxiston & 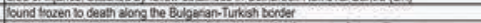 & TimesM \\
\hline $0112: 16$ & $\frac{1}{1}$ & $(18, m 30)$ & Aschanst & Whed alone $\operatorname{lar} 5$ & Mas ont \\
\hline 24146 & 2 & N.N. (ES. Woman: 6.t. & Tukey & 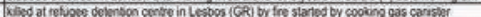 & inderendertives \\
\hline
\end{tabular}




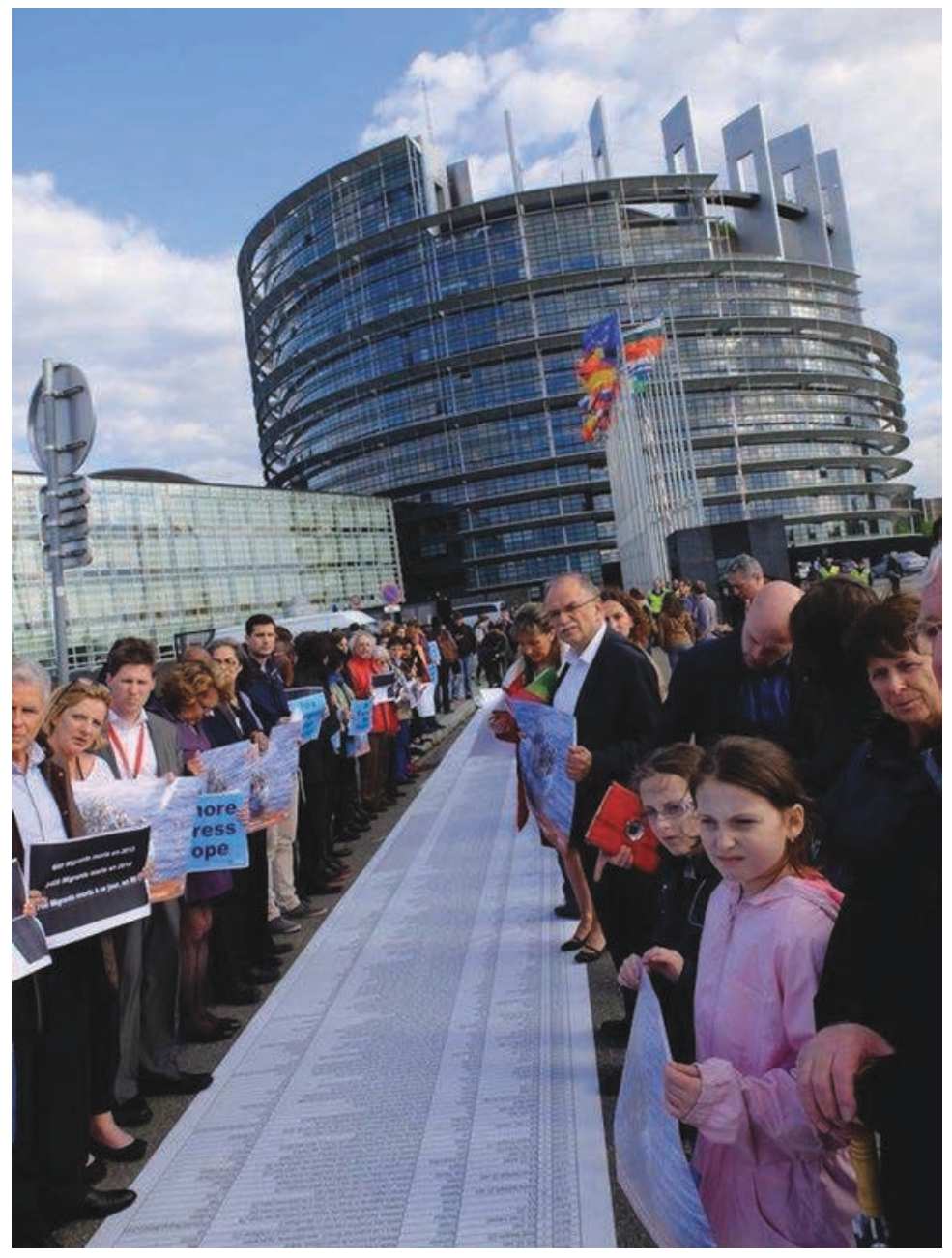

Source : http://unitedagainstrefugeedeaths.eu/about-the-campaign/ about-the-united-list-of-deaths/ 


\section{L'humanitarisation des frontières et le décompte (inter)gouvernemental des morts}

Les décès de migrants sont longtemps demeurés la face sombre du régime migratoire européen ; ils restaient dans l'ombre, alors que le succès d'une politique de contrôle se mesurait en fonction du nombre d'interceptions et de renvois de migrants. Au cours de ces dernières années, cependant, le contrôle des frontières a été habillé d'un langage humanitaire et la prévention des morts aux frontières est devenue une des justifications des politiques en la matière. Ilkka Laitinen, ancien directeur de Frontex, déclarait ainsi, en parlant de I'opération Hera ${ }^{16}$ :

"L'Operation Hera sort du lot. En appliquant des mesures préventives au large des côtes ouest-africaines, Hera a presque complètement tari le flux de migrants irréguliers vers les îles Canaries à travers cette route particulièrement dangereuse. II en a résulté

le sauvetage de centaines, si ce n'est de milliers, de vies. Je crois que cela doit être considéré comme un de nos plus grands succès. $1^{17}$

Cette déclaration est exemplaire de la description de l'acte du contrôle des frontières comme un acte humanitaire, occultant dans le même mouvement le rôle fondamental des politiques migratoires dans le phénomène des morts aux frontières. Ce n'est en effet que parce que le détroit de Gibraltar avait fait l'objet d'une surveillance plus étroite que les migrants se sont dirigés vers les îles Canaries et la route de la côte Atlantique (Carling, 2007). Walters (2011) parle $d^{\prime}$ " humanitarisation des frontières " pour désigner une manière de gouverner les frontières dans un contexte où leur franchissement devient " une question de vie et de mort " ; il montre que cette humanitarisation permet de répondre à une situation nouvelle et dérangeante et de compenser la violence sociale du contrôle des frontières. Si le sauvetage en mer (par les garde-côtes ou d'autres navires) a depuis de nombreuses années constitué le revers humanitaire de l'illégalisation des migrations, la phase actuelle de l'humanitarisation des frontières franchit une nouvelle étape dans ce processus puisqu'elle construit directement le contrôle aux frontières comme un acte humanitaire de sauvetage. La frontière humanitaire rappelle à cet égard le lien inextricable entre violence et soin, caractéristique du pouvoir colonial (Mbembe, 2001).

Cette justification par I'humanitaire du contrôle des frontières a pris une dimension nouvelle à la suite du naufrage du 3 octobre 2013, lors duquel 366 personnes ont perdu la vie alors que leur embarcation se trouvait à moins d'un kilomètre de l'île de Lampedusa. Cet événement a été abondamment commenté et a suscité de vives réactions, de la visite hautement médiatisée du pape François sur cette île (puis, en 2016, sur celle de Lesbos) à l'intérêt d'institutions influentes pour ce sujet (voir Amnesty International, 2014 ; Conseil de I'Europe, 2012 ; Ritaine, 2015). Ce naufrage a également été l'occasion de nombreux discours officiels ; on peut notamment citer celui de Jose Manuel Barroso,

16 L'opération Hera, menée par Frontex en 2006, concernait la migration irrégulière des côtes ouest-africaines vers les îles Canaries. Cf. http://frontex.europa.eu/news/longestfrontex-coordinated-operation-hera-the-canary-islands-WpQlsc (consulté le 27/09/2016).

17 Cf. http://www.eurasylum.org/092010-gen-brig-ilkka-laitinen/ (notre traduction, consulté le 27/09/2016). 
alors président de la Commission européenne, après sa visite à Lampedusa le 8 octobre 2013 : "Nous à la Commission, moi-même et la Commissaire Malmström, nous pensons que l'Union européenne ne peut pas accepter que des milliers de personnes meurent à ses frontières ", déclara-t-il, avant d'annoncer un accroissement du budget de Frontex et le lancement d'Eurosure, un système de surveillance des frontières européen - soit, en d'autres termes, la continuation de l'approche sécuritaire qui a contribué à ces décès ${ }^{18}$.

Quelques jours plus tard, I'Italie lançait I'opération militaire et humanitaire Mare Nostrum, dont l'envergure était sans précédent dans I'histoire récente. Si la lutte contre les passeurs était une dimension importante de cette opération, celle-ci avait également pour mission le sauvetage des migrants : elle représentait ainsi l'archétype de la frontière humanitaire. Même si elle ne pouvait qu'atténuer les effets mortifères du régime de contrôle des frontières (puisque les politiques d'illégalisation des migrations demeuraient en place), elle a tout de même permis, pendant l'année qu'a duré l'opération, de secourir les migrants de manière proactive et par des moyens relativement conséquents.

Le projet Missing Migrants s'inscrit dans ce contexte. Comme l'indique I'OIM, " la recherche derrière ce projet a commencé avec les tragédies d'octobre $2013{ }^{19}$. Alors que l'attention médiatique et publique était à son comble, cette organisation a décidé de se positionner sur ce thème, en constituant notamment sa propre liste de morts aux frontières ${ }^{20}$. L'OIM n'est pas une association, mais une organisation intergouvernementale : en reprenant à son compte l'entreprise de comptage des morts, elle change donc la nature de cette activité. L'OIM se perçoit à la fois comme une source de données fiables et comme un intermédiaire entre différents acteurs. De par sa présence sur le terrain, elle est à même d'avoir accès à des informations, qu'elle peut ensuite compiler pour produire des données globales. De plus, elle entretient des rapports avec un vaste éventail d'acteurs impliqués dans les enjeux migratoires, à commencer par les États et de nombreuses ONG, ce qui lui permet de fonctionner comme une plateforme centralisant des informations d'origines très diverses. L'OIM insiste également sur le contact permanent qu'elle entretient avec les médias, à la fois pour obtenir des informations de la part de journalistes et pour leur fournir des données de qualité. Sur le site Internet du projet, elle affirme en outre être régulièrement contactée par des proches de migrants disparus, qui cherchent des informations sur leur sort ; le site fournit ainsi des adresses électroniques permettant de déposer une demande de ce genre, ou alors d'envoyer des informations à I'OIM. L'organisation aspire donc à un rôle d'intermédiaire, non seulement entre États, société civile et médias, mais aussi entre les victimes et leurs familles. Cette ambition est conforme à un rôle souvent associé aux organisations internationales, à savoir celui d'un intermédiaire fiable et mesuré, dénué d'intérêts propres, et donc capable de parler à tous avec compétence et sans arrière-pensée (honest broker).

18 Déclaration du président de la Commission européenne José Manuel Barroso lors de la Commission européenne du 9 octobre 2013. Cf. http://europa.eu/rapid/press-release SPEECH-13-792_en.htm (notre traduction, consulté le 27/09/2016).

19 Cf. https://missingmigrants.iom.int/about (notre traduction, consulté le 27/09/2016).

20 Notons que le HCR répertorie également depuis 2005 les morts qui lui sont rapportées. Mais cette organisation n'en a pas fait une activité principale et sa base de données n'est pas publiée. 
Alors que les données sur les morts aux frontières étaient brandies par les associations pour formuler une critique des politiques migratoires européennes, I'OIM s'inscrit dans une logique différente. Sa position est explicitée dans un rapport de 2014, intitulé Fatal Journeys. Tracking Lives Lost During Migration (Organisation internationale pour les migrations, 2014). Dans la préface à ce rapport, le directeur général de I'OIM commence par reconnaître, d'une part, qu'il est possible de comprendre pourquoi des migrants prennent le risque de mourir ("Death is a risk worth taking in desperate situations ", Ibid. : 5) et, d'autre part, que si les passeurs ont une part de responsabilité, c'est aussi en raison de politiques migratoires inadéquates que ces décès surviennent (" Limited opportunities for safe and regular migration drives would-be migrants into the hands of smugglers ", Ibid.). Mais l'argumentaire se fait ensuite extrêmement prudent ; I'OIM ne traite que de la manière de compter les morts, jamais des façons de les prévenir : "While views may differ on how best to limit the number of migrant deaths, there is a broad agreement on the need for better data " (Ibid. : 17). L'OIM constate l'existence d'interprétations divergentes sur les mécanismes qui conduisent à ces décès; sans prendre réellement position, elle se contente d'affirmer le besoin de données - l'objectif étant alors pour l'OIM d'occuper une position centrale dans ce domaine. Alors que le compte des morts était pour les acteurs de la société civile la base empirique pour demander une réorientation des politiques migratoires, ces revendications politiques ont largement disparu du discours de I'OIM.

L'OIM liste trois motivations à sa démarche (Ibid. : 34-36). D'abord, les données chiffrées sur les morts aux frontières permettent d'inciter les États à se préoccuper de cette situation : sans préjuger de ce que sera leur réaction, I'OIM souhaite seulement qu'ils s'en préoccupent. Cela est en accord avec sa position d'acteur intergouvernemental, soucieux de respecter la souveraineté de ses États membres. Ensuite, les données permettent de comprendre le " profil " des personnes à risque, ce qui facilite la prévention de ces décès, tout en permettant de sensibiliser les migrants potentiels avant leur départ : "The information provided by survivors and the families of those who die can also help to persuade others not to embark on such risky journeys " (Ibid. : 35). On reconnaît là une préoccupation classique de I'OIM, qui a de longue date lancé des campagnes d'informations destinées à informer - c'est-à-dire, le plus souvent, à décourager - les migrants (Nieuwenhuys et Pécoud, 2008 ; Heller, 2014). Enfin, I'OIM avance un argument d'ordre moral, centré sur la dignité des défunts et la nécessité de fournir à leur famille toutes les informations possibles.

Aux yeux de l'OIM, la sensibilité politique des données sur les morts aux frontières milite en faveur de son propre rôle. Les États, soucieux d'éviter que ces chiffres n'alimentent une critique de leur politique, seraient naturellement peu enclins à coopérer avec les ONG pour compter les morts. Le résultat est que ces données sont mal collectées, faute de coopération : "National authorities have not given priority to collecting this data, given that the migrant death count is often perceived by civil society groups as an indication of the consequences of tougher border control "( Ibid. : 36). Cette défiance entre États et société civile légitime l'intervention de I'OIM, qui aspire ainsi à se placer au-dessus de la mêlée. Face à un enjeu clivant, elle souhaite dépasser les antagonismes et faire autorité, en produisant les statistiques les plus sérieuses - mais sans pour autant adopter une position politique qui la placerait dans un camp ou dans 
un autre. On perçoit là une tendance lourde au sein des Ol : ces dernières sont avides de chiffres et en produisent des quantités impressionnantes, sur tous les sujets possibles; cette production statistique s'accompagne cependant d'une prudence politique, qui conduit les Ol à éviter de prendre ouvertement position sur des sujets sensibles (Bréant, 2012 ; Pécoud, 2015).

L'OIM dispose de moyens sensiblement supérieurs à ceux des ONG. Cela se manifeste dans une mise à jour très régulière du site Internet du projet, ainsi que dans la pléthore de graphiques et de cartes disponibles : il est possible d'y trouver les données par jour/mois/année, par pays d'origine des défunts, par le lieu du décès, en croisant si nécessaire ces informations. Des cartes sont réalisées par pays, et représentent les routes migratoires empruntées par les migrants aux quatre coins de la planète. Un certain nombre de cartes interactives constituent probablement le support le plus sophistiqué dans la présentation de ces données : sur la carte de l'Europe et de ses environs apparaissent différentes informations, comme les routes migratoires, les réfugiés, les déplacés internes ou les " tendances récentes ". L'ensemble est facile d'accès, aisément téléchargeable pour quiconque s'intéresse à la question, et se présente sous un jour scientifique et cartographique de bonne facture.

L'OIM a ainsi repris une démarche initiée par des associations. Si on compare ses statistiques à celles de la société civile, on note également de nombreuses ressemblances : la liste de I'OIM détaillant le lieu et la nature du décès fait immédiatement penser à la liste d'United for Intercultural Action, tandis que le recours à l'outil cartographique s'inspire visiblement des cartes réalisées par $L e$ Monde Diplomatique. De ce point de vue, les ONG ont bel et bien inventé une démarche qui fait référence, et qui inspire aujourd'hui l'initiative de I'OIM. Mais il y a également des différences, en particulier en ce qui concerne la définition du phénomène.

L'OIM est nettement plus restrictive quant aux cas de décès considérés : elle se concentre sur les migrants qui sont " morts ou ont disparus aux frontières extérieures des États, ou dans le processus de migration vers une destination internationale " et ne compte donc pas les morts survenues dans des centres de détentions, pendant des renvois ou expulsions, ou liées au statut irrégulier des migrants; elle ne compte pas non plus les morts résultant de l'exploitation des migrants sur le marché du travail, et ce alors que ces morts pourraient être attribuées aux effets du contrôle des frontières et des politiques migratoires ${ }^{21}$. II en résulte que peu de cas de décès sont comptés sur le sol européen ${ }^{22}$; la vaste majorité des cas concerne la Méditerranée ${ }^{23}$ et I'Afrique ${ }^{24}$. Cela conduit I'OIM à ne pas compter les morts qui ont lieu lorsque les migrants sont sous le contrôle direct des États, ce qui déboucherait sur une critique à l'égard des gouvernements. Cela s'inscrit dans la continuité de la stratégie de I'OIM, qui a toujours été extrêmement réticente à critiquer ses États membres. Elle se distingue à cet égard d'autres organisations internationales qui, à l'instar du HCR, émettent

\footnotetext{
21 Cf. http://missingmigrants.iom.int/methodology (consulté le 28/11/2017).

22 Cf. https://missingmigrants.iom.int/region/europe (consulté le 28/11/2017).

23 Cf. https://missingmigrants.iom.int/region/mediterranean (consulté le 28/11/2017).

24 Cf. https://missingmigrants.iom.int/region/africa (consulté le 28/11/2017).
} 


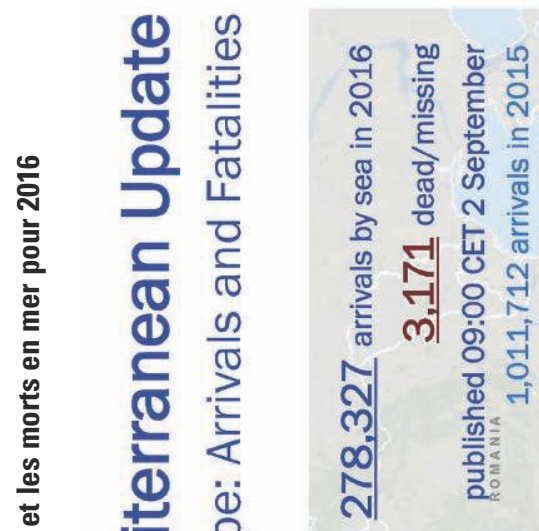

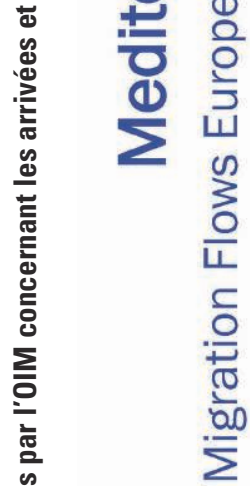

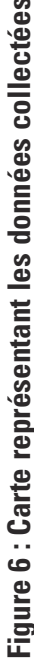


régulièrement des réserves lorsqu'elles perçoivent des risques de non-respect des principes qu'elles sont chargées de faire respecter. Les statistiques de I'OIM sont donc aseptisées, c'est-à-dire débarrassées des éléments qui pourraient directement remettre en cause les pratiques des États. À certaines occasions, I'OIM va même jusqu'à les féliciter ; ainsi, annonçant en juin 2016 que près de 2900 migrants sont morts en tentant de traverser la Méditerranée au cours du premier semestre 2016, I'OIM déclarait à la presse : "L'Europe a fait un remarquable travail, des milliers de vies ont été sauvées rien que cette année. Mais près de 3000 personnes sont mortes, ce qui signifie que l'Europe n'entreprend pas tout ce qu'il est possible de faire ${ }^{25}$.

En résumé, l'OIM témoigne d'une professionnalisation et d'une centralisation de la collecte de données sur les morts aux frontières, mais également d'une dépolitisation du discours qui les accompagne. Face à l'arrivée de l'OIM sur "leur " terrain, quelles sont les implications et quelle peut-être la position des acteurs issus de la défense des droits des migrants ? D'un côté, elle peut y voir le succès d'un travail de longue haleine : après des années de travail indépendant, souvent bénévole et avec peu de soutien, la société civile est parvenue à imposer la réalité de ces morts à un large public, au point que des OI ont repris cette activité à leur compte. Mais en devenant un acteur incontournable de ces statistiques, I'OIM a en partie privé la société civile d'un outil privilégié pour véhiculer leur discours critique.

Ce n'est pas là un cas isolé. Boli etThomas (1999) montrent que de nombreux enjeux politiques ont été soulevés par la société civile, avant d'être repris par des OI, puis - en dernier lieu - par les États. La société civile est à l'origine de ce qu'ils appellent des " scripts ", c'est-à-dire des manières d'aborder la réalité et de traiter les problèmes sociaux et politiques. Ces scripts (qu'on pourrait aussi qualifier de "cadres " ou de " paradigmes") sont repris par les Ol, dans le cadre de leur coopération avec la société civile. À leur tour, les Ol sont en mesure $\mathrm{d}^{\prime}$ inciter les États à les reprendre à leur compte ${ }^{26}$. Si on suit ce raisonnement, le comptage des morts aux frontières serait au milieu de ce processus : né dans la société civile, il serait actuellement repris par une Ol, avant - peut-être - de devenir une nouvelle norme pour les États. Il est donc possible d'envisager que, de la même manière qu'ils comptent les migrants vivants qui entrent sur leur territoire, les États en viennent également à compter les migrants morts, et que cela devienne une nouvelle norme statistique.

Dans l'attente de la réalisation (ou non) de cette éventualité, il reste que la société civile peut maintenant s'interroger sur ce qui différencie sa pratique de celle de I'OIM. Certes, des différences importantes demeurent, en particulier quant à la définition des « morts aux frontières ». Mais il n'en demeure pas moins

25 Propos rapportés par Reuters (2016) Le nombre de migrants morts en Méditerranée atteint un record, Reuters, 01 juillet 2016, [en ligne] consulté le 27/09/2016. URL : http:// fr.reuters.com/article/topNews/idFRKCNOZH4UQ

26 Boli et Thomas donnent de nombreux exemples. C'est ainsi la société civile qui est à l'origine de la prise de conscience du changement climatique et de la nécessité de protéger l'environnement ; I'ONU a repris cette idée et tous les États sont aujourd'hui engagés dans ce domaine. De même, les droits des femmes ont été "inventés " par des organisations militantes, avant de faire l'objet de campagnes internationales par les Ol, qui font aujourd'hui pression sur tous les pays du monde pour qu'ils adoptent des stratégies d'égalité homme femme. 
que la pratique de la société civile a perdu une part de son " mordant " : alors que ses contres statistiques transgressaient la frontière de l'attention politique et publique, cette dimension transgressive a clairement été réduite par le fait que ces statistiques sont maintenant produites par une $\mathrm{Ol}$, et que le discours sur les morts est devenu un élément central de la justification même du contrôle des frontières. Comme discuté plus haut, cette situation témoigne des interactions entre pouvoir et résistance, engagés dans un corps à corps dans lequel les deux logiques ne sont jamais figées, mais se forment au contraire mutuellement dans un mouvement dialectique.

\section{(Re)compter pour demander des comptes aux États}

Pour la société civile, le compte des morts aux frontières fondait la revendication de rendre les États comptables de celles-ci. Ce lien entre compte et comptabilité (accountability en anglais) est menacé par l'intervention de I'OIM et cette dernière section aborde donc la manière dont tant les chercheurs que la société civile peuvent tenter de renouer ce lien, c'est-à-dire parvenir à repolitiser les chiffres. Nous nous penchons sur une tentative de ce type, à savoir le rapport Death by Rescue. The Lethal Effects of the EU's Policies of Non-Assistance, produit par des chercheurs de l'Université de Londres (dont l'un des auteurs de cet article $)^{27}$. Ce rapport analyse l'impact des décisions prises par l'UE et les États européens - ici l'arrêt de l'opération Mare Nostrum et son (non-)remplacement par I'opération plus limitée Triton (menée par Frontex) en automne 2014 - sur le danger de la traversée pour les migrants.

Le rapport reconstruit d'abord les changements politiques à l'œuvre entre 2014 et début 2015. Alors que l'opération italienne Mare Nostrum, dont les navires étaient déployés à proximité des côtes libyennes pour secourir les migrants de manière proactive, était décriée comme créant un "appel d'air ", les décideurs européens ont pris la décision de ne pas soutenir sa continuation sous une forme européanisée, et de la remplacer par l'opération Triton de Frontex. Or celle-ci fut dotée de moyens plus limités, confinée à une zone opérationnelle plus proche des côtes européennes, et conçue sur la base d'un objectif de contrôle des frontières (en opposition à l'opération Mare Nostrum, davantage axée sur le sauvetage). Les décideurs européens avaient pourtant été informés - par le biais de nombreuses ONG, d'Ol, mais aussi de Frontex - que ce changement rendrait la traversée plus périlleuse pour les migrants, et aurait pour conséquence probable un accroissement du nombre de morts en mer.

Sur la base d'une analyse de plusieurs cas de naufrages qui ont eu lieu à la suite de ce changement de politique, ainsi que d'une analyse statistique de la mortalité, le rapport démontre que le danger de la traversée a effectivement augmenté à la suite de cette décision, et que cette augmentation est largement imputable à celle-ci. La mesure de la mortalité est basée sur les statistiques concernant les arrivées sur les côtes européennes (compilées par le HCR) et sur les statistiques sur les morts en mer (produites par I'OIM) : la mortalité consiste dans le rapport entre le nombre de personnes ayant tenté la traversée et celles finalement arrivées. Pour une période identique, entre janvier et avril, le

27 Cf. https://deathbyrescue.org/ (consulté le 27/09/2016). 


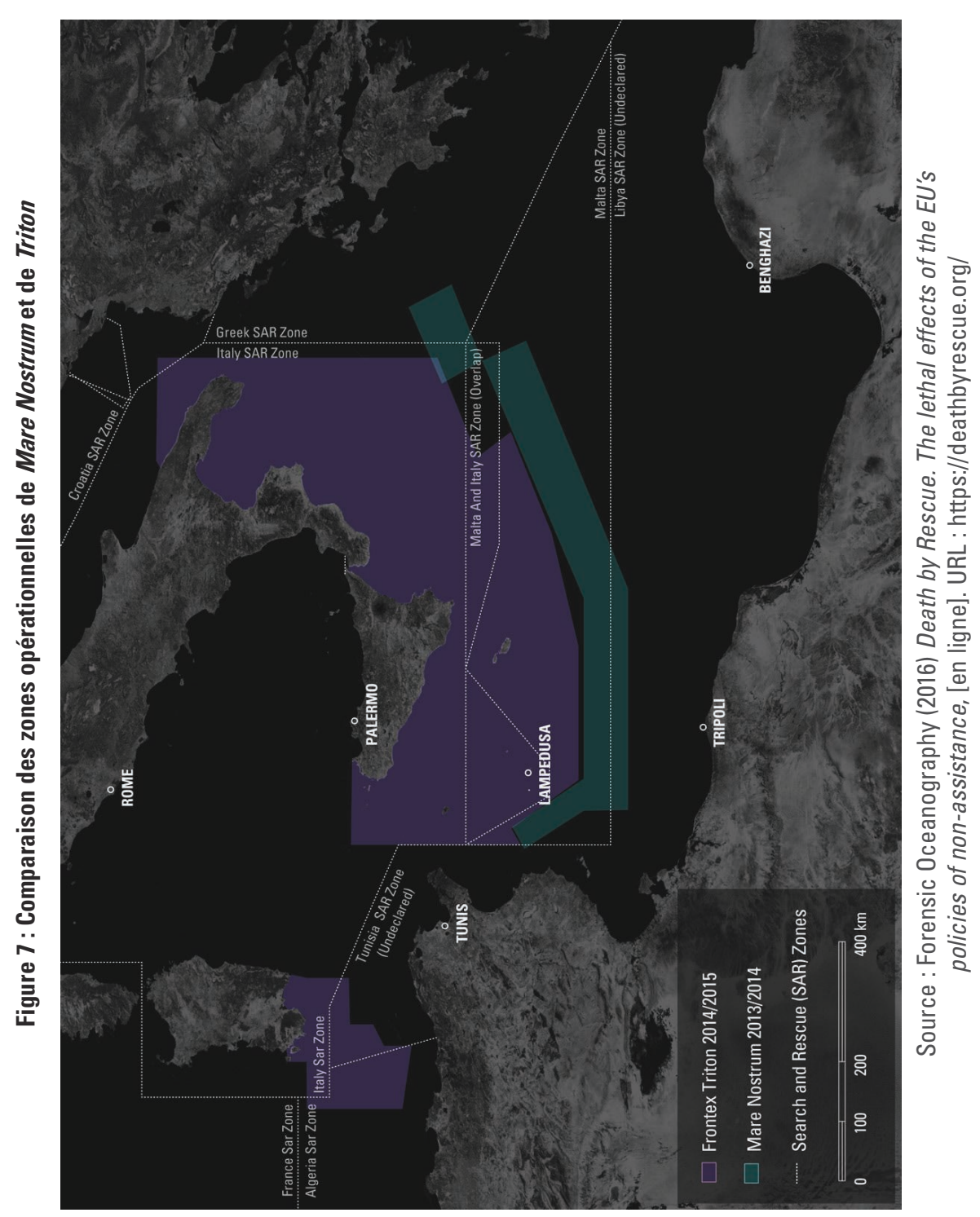


Figure 8 : La mesure de la mortalité calculée pour la Méditerranée centrale sur la base des données HCR (arrivées) et OIM (morts)

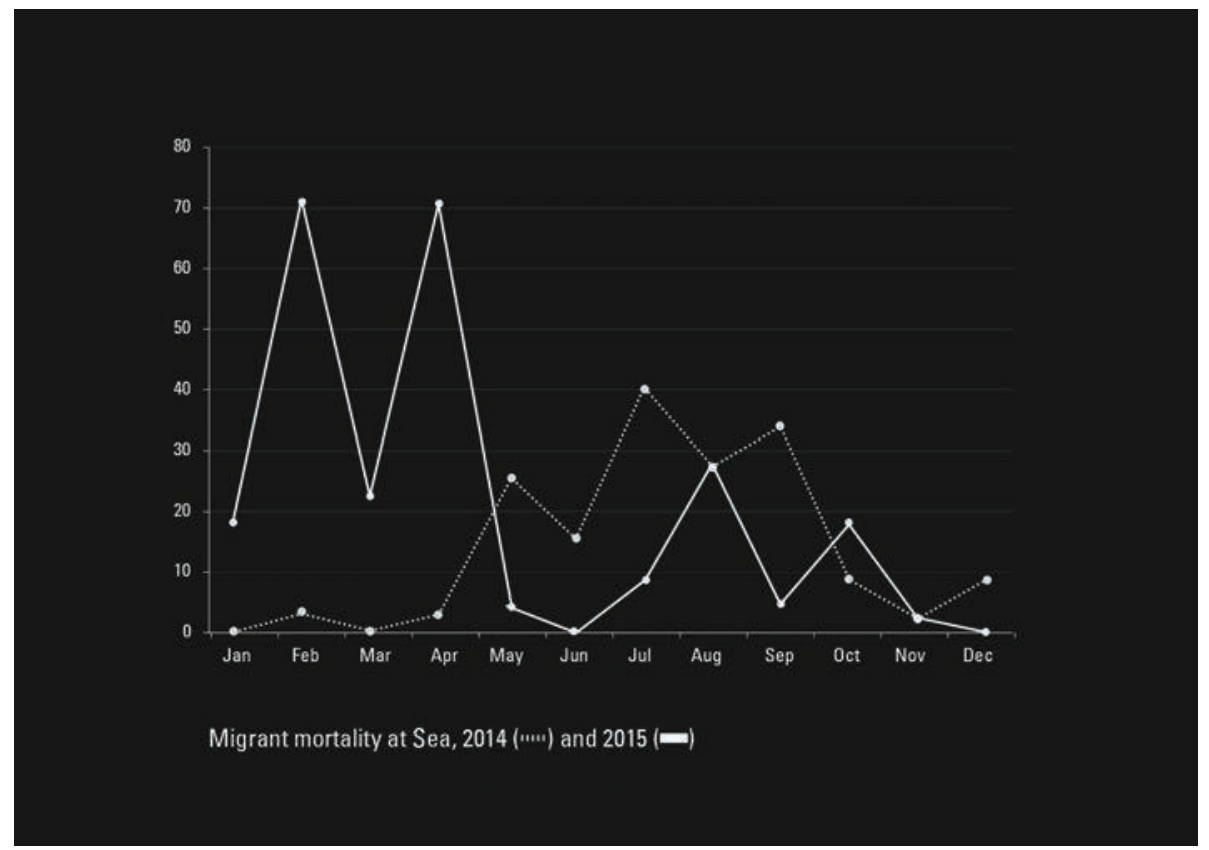

Source : Forensic Oceanography (2016) Death by Rescue. The lethal effects of the EU's policies of non-assistance, [en ligne]. URL : https://deathbyrescue.org/ 
nombre de personnes traversant la mer était sensiblement le même (26 644 en 2014, 26228 en 2015), mais le nombre de morts est passé de soixante à 1687 : la mortalité s'en trouvait ainsi multipliée par vingt-sept. La mortalité retomba à la suite de deux naufrages importants en avril 2015, lesquels entrainèrent le redéploiement de moyens conséquents par les États et les ONG humanitaires. Dans la mesure où les autres facteurs affectant le danger de la traversée étaient demeurés stables, le rapport conclut que cette augmentation était principalement imputable aux effets de la décision politique de l'UE et de ses États membres.

La démarche des auteurs du rapport Death by Rescue ne cherche donc pas à produire de nouvelles données et de nouveaux chiffres. Elle aspire plutôt à s'approprier les statistiques compilées par deux OI, afin de les repolitiser. La qualité et la crédibilité de ces données sont à cet égard un atout, car elles permettent d'arriver à des conclusions d'autant plus étayées. La stratégie est ici différente de celles précédemment suivies par la société civile : là où les associations produisaient des chiffres pour contester les politiques migratoires, ce rapport souhaite atteindre le même objectif contestataire, mais en " retournant " les chiffres produits par les $\mathrm{Ol}$ - et donc en contrant la dépolitisation des chiffres par les OI.

\section{Conclusion}

Les statistiques sur les morts aux frontières sont devenues un champ de bataille des politiques migratoires. Ce nouveau terrain de lutte illustre deux tendances importantes : d'une part, I'humanitarisation des frontières voit le comptage de ces morts repris par les acteurs mêmes du contrôle des frontières, au nom de la protection des migrants ; d'autre part, de par cette ambivalence sémantique de la souffrance des migrants, les discours et les pratiques de la société civile, des États et des Ol tendent à ne plus être nettement séparables les uns des autres et à opérer dans un champ mixte où discours et pratiques circulent d'un bord à l'autre.

Alors que pour la société civile le comptage des morts est justifié d'une part par l'exigence morale de donner un nom, une identité, voire une sépulture, à ces morts, et d'autre part par la volonté de mettre les États face aux conséquences de leurs politiques, la dénonciation est absente du discours de I'OIM, qui se positionne comme acteur neutre fournissant "simplement " des données permettant en retour de répondre au phénomène documenté. Les recommandations de I'OIM se limitent ainsi à I'harmonisation de la collecte des données sur les morts en mer, et éventuellement à la proposition de mesures permettant de diminuer le nombre de morts et de "sauver des vies ", sans remise en cause fondamentale des logiques des politiques migratoires. À cet égard, I'initiative de I'OIM apparaît comme une tentative de neutraliser les chiffres, de les rendre inoffensifs, de réduire la menace qu'ils représentent pour l'ordre étatique - voire de les récupérer pour renforcer cet ordre. Mais si l'OIM s'est approprié une pratique issue de la société civile en la dépolitisant, rien n'empêche la société civile ou les chercheurs de se saisir de ces données à leur tour et de leur réinsuffler un sens nouveau en liant à nouveau l'acte de compter et la demande de comptes aux États. 


\section{Références bibliographiques}

Agier Michel (2008) Gérer les indésirables. Des camps de réfugiés au gouvernement humanitaire, Paris, Flammarion, $349 \mathrm{p}$.

Albahari Maurizio (2015) Crimes of Peace. Mediterranean Migrations at the World's Deadliest Border, Philadelphia, University of Pennsylvania Press, 288 p.

Amnesty International (2014) The Human Cost of Fortress Europe. Human Rights Violations against Migrants and Refugees at Europe's Borders, London, Amnesty International, $54 \mathrm{p}$.

Andrijasevic Rutvica et Walters William (2011) L'Organisation international pour les migrations et le gouvernement international des frontières, Cultures \& Conflits, 84, pp. 13-43.

Blackburn Robin (2011) The American Crucible: Slavery, Emancipation and Human Rights, London, Verso, 520 p.

Blanchard Emmanuel, Clochard Olivier et Rodier Claire (2008) Compter les morts, Plein Droit, 2 (77), pp. 30-34.

Boli John and Thomas Georges M. (1999) Constructing World Culture: International Nongovernmental Organizations since 1875, Stanford, Stanford University Press, $378 \mathrm{p}$.

Brachet Julien (2016) Policing the Desert: The IOM in Libya Beyond War and Peace, Antipode, 48 (2), pp. 272-292.

Bréant Hugo (2012) Démontrer le rôle positif des migrations internationales par les chiffres. Une analyse de la rhétorique institutionnelle du système des Nations unies, Mots. Les langages du politique, 100, pp. 153-171.

Bruno Isabelle, Didier Emmanuel et Prévieux Julien (Éds.) (2014) Statactivisme. Comment lutter avec des nombres, Paris, Zones/La Découverte, 208 p.

Carling Jørgen (2007) Migration Control and Migrant Fatalities at the SpanishAfrican Borders, International Migration Review, 41 (2), pp. 316-343.

Clochard Olivier et Lambert Nicolas (2015) L'évolution d'un régime. Mort aux frontières et contrôles migratoires en mer Méditerranée, in Camille Schmoll, Hélène Thiollet et Catherine Wihtol de Wenden Éds., Migrations en Méditerranée. Permanence et mutations à l'heure des révolutions et des crises, Paris, CNRS Éditions, pp. 145-156.

Conseil de l'Europe (2012) Lives lost in the Mediterranean Sea: Who is responsible?, Strasbourg, Conseil de l'Europe, $24 \mathrm{p}$.

Cuttitta Paolo (2014) From the Cap Anamur to Mare Nostrum: Humanitarianism and Migration Controls at the EU's Maritime Borders, in Claudio Matera et Amanda Taylor Eds., The Common European Asylum System and Human Rights: Enhancing Protection in Times of Emergencies, La Haye, Asser Institute, pp. 21-29.

De Genova Nicholas (2013) Spectacles of migrant "illegality": The scene of exclusion, the obscene of inclusion, Ethnic and Racial Studies, 36 (7), pp. 11801198.

Foucault Michel (2004) Sécurité, Territoire, Population, Paris, EHESS/Gallimard/ Seuil, 448 p. 
Georgi Fabian (2010) For the Benefit of Some:The International Organization for Migration and its Global Migration Management, in Martin Geiger and Antoine Pécoud Eds., The Politics of International Migration Management, Basingstoke, Palgrave, pp. 45-72.

Heller Charles (2015) Liquid Trajectories: Documenting Illegalised Migration and the Violence of Borders, Doctoral Thesis, Centre for Research Architecture, Department of Visual Cultures, Goldsmiths University of London, 283 p.

Heller Charles (2014) Perception management. Deterring potential migrants through information campaigns, Global Media and Communication, 10 (3), pp. 303-318.

Heller Charles et Pezzani Lorenzo (2014) Traces liquides : Enquête sur la mort de migrants dans la zone-frontière maritime de l'Union européenne, Revue Européenne des Migrations Internationales, 30 (3-4), pp. 71-107.

Kobelinsky Carolina (2015) Les morts aux frontières de I'Espagne. Trajectoire des corps et pratiques locales, in Camille Schmoll, Hélène Thiollet et Catherine Wihtol de Wenden Éds., Migrations en Méditerranée. Permanence et mutations à I'heure des révolutions et des crises, Paris, CNRS Éditions, pp. 191-201.

Legg Stephen (2005) Foucault's Population Geographies: Classifications, Biopolitics and Governmental Spaces, Population Space Place, 11 (3), pp. 137-156.

Mbembe Achille (2001) De la postcolonie. Essai sur l'imagination politique dans l'Afrique contemporaine, Paris, Karthala, 293 p.

Migreurop (2012) Atlas des migrants en Europe. Géographie critique des politiques migratoires européennes, Paris, Armand Colin, 144 p.

Nevins Joseph (2003) Thinking Out of Bounds: A Critical Analysis of Academic and Human Rights Writings on Migrant Deaths in the U.S.-Mexico Border Region, Migraciones Internacionales, 2 (2), pp. 171-190.

Newman David (2006) The lines that continue to separate us: Borders in our "borderless" world, Progress in Human Geography, 30, pp. 143-161.

Nieuwenhuys Céline et Pécoud Antoine (2008) Campagnes d'information et traite des êtres humains à l'est de l'Europe, Espace Populations Sociétés, 2, pp. 319-330.

Organisation internationale pour les migrations (2014) Fatal Journeys. Tracking Lives Lost During Migration, Genève, OIM, $216 \mathrm{p}$.

Pécoud Antoine (2017) Entre " gestion " et contrôle des migrations. Discours et pratiques de l'Organisation internationale pour les migrations, Critique internationale, 76, pp. 81-99.

Pécoud Antoine (2015) Depoliticising Migration. Global Governance and International Migration Narratives, Basingstoke, Palgrave, 156 p.

Perry Amanda T. (2012) A Traffic in Numbers: The Ethics, Effects, and Affect of Mortality Statistics in the British Abolition Debates, Journal for Early Modern Cultural Studies, 2 (4), pp. 78-104.

Pezzani Lorenzo (2015) Liquid traces: Spatial practices, aesthetics and humanitarian dilemmas at the maritime borders of the EU, Doctoral Thesis, Centre for Research Architecture, Department of Visual Cultures, Goldsmiths University of London, 278 p. 
Ritaine Évelyne (2015) Quand les morts de Lampedusa entrent en politique : damnation memoriae, Cultures \& Conflits, 99-100, pp. 117-142.

Rose Nikolas (1999) Powers of Freedom: Reframing PoliticalThought, Cambridge: Cambridge University Press, $334 \mathrm{p}$.

Rose-Redwood Reuben (2012) With Numbers in Place: Security, Territory, and the Production of Calculable Space, Annals of the Association of American Geographers, 102 (2), pp. 295-319.

Scott James C. (1998) Seeing Like a State: How Certain Schemes to Improve the Human Condition Have Failed, Yale, Yale University Press, 460 p.

Stenum Helle (2012) Making Migrants Governable: Counting and defining the “illegal” migrant, Journal of Nordic Migration Research, 2 (4), pp. 280-288.

Tazzioli Martina (2015) The politics of counting and the scene of rescue. Border deaths in the Mediterranean, Radical Philosophy, 192, pp. 3-6.

Van Houtum Henk and Boedeltje Freerk (2009) Europe's Shame: Death at the Borders of the EU, Antipode, 41 (2), pp. 226-230.

Walters William (2011) Foucault and frontiers: Notes on the birth of the humanitarian border, in Ulrich Bröckling, Susanne Krasmann et Thomas Lemke Eds., Governmentality: Current Issues and Future Challenges, New York, Routledge, pp. $138-164$.

Weber Leanne and Pickering Sharon (2011) Globalization and Borders. Death at the Global Frontier, Basingstoke, Palgrave, 262 p. 


\section{Charles Heller et Antoine Pécoud}

\section{Compter les morts aux frontières : des contre-statistiques de la société civile à la récupération (inter)gouvernementale}

Cet article analyse la production de statistiques sur le phénomène des " morts aux frontières " dans la région euro-méditerranéenne, dans un contexte de crise des réfugiés qui voit des milliers de migrants mourir en tentant d'atteindre l'Europe. Ce travail de documentation et de collecte de données a été réalisé, depuis le début des années 1990, par la société civile et les associations de défense des migrants, dans le but de dénoncer le coût humain des politiques européennes de contrôle des frontières. Depuis 2013 cependant, I'Organisation internationale pour les migrations (OIM) produit également des statistiques à ce sujet (projet Missing Migrant). L'implication d'un acteur intergouvernemental dans un champ initialement occupé par les ONG change la nature et le sens politique de ces statistiques, d'une part parce que I'OIM joue elle-même un rôle important dans les politiques de contrôle des pays occidentaux et contribue donc à alimenter les prises de risques et les décès de migrants, et d'autre part parce cette organisation a pour principe de ne jamais critiquer ses États-membres, et tente donc de dépolitiser les chiffres sur les morts aux frontières en les isolant du contexte qui conduit à ce phénomène. Cet article analyse les raisons qui poussent I'OIM à reprendre à son compte ce comptage initié par la société civile et la manière dont cette activité s'insère dans ses stratégies et ses discours. II interroge finalement les conséquences de l'implication de I'OIM pour la société civile et la manière dont il est possible de repolitiser cette activité statistique.

\section{Counting Migrant Deaths at the Borders: From Civil Society's Counter-statistics to (Inter)governmental Recuperation}

This article analyses the production of statistics on "deaths at the border" in the euro-Mediterranean region, in a context of crisis marked by the deaths of thousands of migrants trying to reach Europe. Statistics and data collection on this issue have, since the early nineties, been carried out by civil society groups and NGOs working on defending migrants' rights, with the purpose of denouncing the human cost of border control and of European immigration policies. Since 2013, however, the International Organization for Migration (IOM) has started to produce its own data on the topic as part of its Missing Migrants Project. The implication of an intergovernmental organization in a field traditionally associated with civil society changes the political meaning of these statistics, first because IOM is also involved in assisting European states in controlling migration, and therefore in creating the conditions that incite migrants to take often deadly risks, and second because this organization never criticizes its member-states and therefore attempts to depoliticize migrant deaths by isolating them from their context. This article analyses the reasons behind IOM's counting of migrant deaths, and the way this activity fits into its strategies and discourses. It questions the ways in which civil society can react to this new context, and repoliticise the statistics on migrant deaths. 


\section{Contabilizar los muertos en las fronteras: de las contra-estadísticas de la sociedad civil a la recuperación (inter)gubernamental}

Este artículo analiza la producción de estadísticas sobre el fenómeno de las "muertes en las fronteras» en la región euromediterránea, en un contexto de crisis de los refugiados que ve a millares de migrantes morir intentando alcanzar Europa. Este trabajo de documentación y de recogida de datos fue realizado, desde el principio de los años noventa, por la sociedad civil y las asociaciones de defensa de los migrantes, con el fin de denunciar el coste humano de las políticas europeas de control de fronteras. Desde 2013 sin embargo, la Organización internacional para las migraciones (OIM) ha producido también estadísticas a este respecto (proyecto Missing Migrant). La implicación de un actor intergubernamental en un campo inicialmente ocupado por las ONG cambia la naturaleza y el sentido político de estas estadísticas, por una parte porque la OIM desempeña ella misma un papel importante en las políticas de control de los países occidentales y contribuye por tanto en cierto modo a incrementar los riesgos y las muertes de migrantes, y por otra parte porque esta organización tiene por principio de omitir toda crítica hacia sus Estados miembros, intentando por tanto despolitizar las cifras sobre las muertes en las fronteras separándolas del contexto que conduce a este fenómeno. Este artículo analiza las razones que impulsan a la OIM a asumir este recuento iniciado por la sociedad civil y la manera en que esta actividad se inserta en sus estrategias y sus posiciones. El texto interroga finalmente las consecuencias de la implicación de la OIM para la sociedad civil y la manera que sería posible para repolitizar esta actividad estadística. 